\title{
Publisher Correction: HOS1 activates DNA repair systems to enhance plant thermotolerance
}

Shin-Hee Han (1), Young-Joon Park and Chung-Mo Park (1)

Correction to: Nature Plants https://doi.org/10.1038/s41477-020-00809-6, published online 16 November 2020.

In the version of this Letter originally published, the boxplot in the lower-right corner of Fig. 1 had a typo in its key. The label for the light-grey shaded bars was incorrect as 'hos1-5 35S:mHOS1'; it should have been 'hos1-3 35S:mHOS1'. This has now been corrected.

Published online: 21 December 2020

https://doi.org/10.1038/s41477-020-00842-5

(C) The Author(s), under exclusive licence to Springer Nature Limited 2020 